\title{
A Collaborative Services Ecosystem for Ambient Assisted Living
}

\author{
Luis M. Camarinha-Matos, João Rosas, Ana Inês Oliveira, and Filipa Ferrada \\ Universidade Nova de Lisboa, Faculty of Sciences and Technology \\ Campus de Caparica, 2829-516 Monte Caparica, Portugal \\ cameuninova.pt
}

\begin{abstract}
A conceptual architecture for ambient assisted living is introduced as a contribution to the development of an ecosystem of products and services supporting active ageing. In order to facilitate understanding and better interrelate concepts, a 3-layered model is adopted: Infrastructure layer, Care and assistance services layer, and AAL ecosystem layer. A holistic perspective of ambient assisted living, namely considering four important life settings is adopted: (i) Independent living; (ii) Health and care in life; (iii) Occupation in life; and (iv) Recreation in life.
\end{abstract}

Keywords: Collaborative networks, Services ecosystem, ICT and Ageing.

\section{Introduction}

The severe demographic changes faced by most developed countries, leading to a rapid increase of the percentage of aged population, raises tough challenges to our society. In this context there is an urgent need to find effective and affordable solutions to provide care and assistance to elderly.

Technology, and particularly high-speed pervasive broadband connectivity, cloudcomputing and web-based technologies, offer new opportunities to provide care and assistance, as well as new ways of working, facilitate social interaction, and reduce limitations imposed by location and time. Many research projects and pilot experiments have focused on ICT and ageing (see, for instance, [1], [2], [3], [4]).

But many good ideas and promising pilot cases fail to scale because the adopted approaches have been excessively techno-centric. A purely technology centered approach, without consideration of the socio-organizational aspects is likely to add only marginal value, not getting accepted by users, or not finding a sustainable business approach for wider deployment. Therefore, while designing a new conceptual architecture for ICT and Ageing it is fundamental to also address the need for organizational and cultural change.

On the other hand, the frequent association of senior citizens with a dependent stage of life does no longer match the reality. The adoption of the concept of "active ageing" provides a more appropriate understanding of the later phases of life [5]. Furthermore, the notion of "productive ageing" [6] has opened new perspectives for a change in the way society often perceives older people. Thus supporting the active 
ageing process is not only about creating an environment exclusively focused on providing healthcare and assistance but rather a more comprehensive one, in which the elderly citizens do not feel excluded, and have a chance to use their knowledge and expertise in a fruitful way, by making a valued contribution to the communities in which they live [7], [8], [9].

Aiming at providing a contribution to the ICT and Ageing area, the Portuguese AAL4ALL project is focused on the development of an ecosystem of products and services for Ambient Assisted Living (AAL), complemented with an adequate business model for this ecosystem. The ALL4ALL consortium involves 32 partners from industry, service providers, and academia, associated to the Health Cluster Portugal.

The underlying assumption in this project is that the creation of effective support environments for the ageing citizens requires the involvement and effective coordination of multiple stakeholders, from diverse sectors and distinct backgrounds. Hence, before addressing specific (technical) implementation approaches and technologies, it is important to consolidate concepts in order to mobilize and align all the needed stakeholders. As such, one of the initial results of the project was the establishment of a conceptual architecture for AAL, which is summarized in this paper. The aim is not simply to support the development of (complex) technological artifacts, but rather conceive systems to support the formation and operation of sustainable AAL ecosystems.

\section{Trends in Elderly Care Services}

Past research and developments in elderly care services as well as current market offers are characterized by some fragmentation. The focus has been predominately put on the development of isolated services - e.g. monitoring of some health related parameter, fall detection, agenda reminder, alarm button, etc. - each one typically provided by a single organization, and often showing an excessive techno-centric flavor. A current trend is to move from fragmented services to progressively more integrated care services [9], [10], which are likely to be provided by multiple stakeholders through well-elaborated collaboration mechanisms. Furthermore, the importance of the role of communities and other forms of collaborative networks involving all stakeholders, operating as an ecosystem, is being recognized.

At this point, we should note that a term frequently causing misunderstandings is what refers to the concept of service, which is used with different meanings by different communities. Therefore, we distinguish two types of services:

- Software services - basically software functionalities that are (remotely) accessible or callable (e.g. web services). This concept corresponds to the view of service typically adopted by ICT experts.

- Care and Assistance Services - which correspond to the services provided to the end users (senior citizens, in this case). This notion is equivalent to what is usually called business services. A care and assistance service may involve a number of software services and human intervention. The actual structure of such service also depends on the interaction between the provider and the user, and may ultimately (and dynamically) vary according to the flow of that interaction. 
Associated to the notion of service - either software service or care and assistance service - there is the notion of service provider. Since a provider might offer more than one service, it is convenient to introduce the concept of service entity - an encapsulation of the various services provided by the same entity; in other words, a representation of a service provider [9], [10]. For instance, a device used in AAL can be represented (modeled) as a service entity that provides several software services (the software functionalities of the device). Similarly, a care institution can be represented by a service entity encapsulating all care and assistance services provided by that institution.

On the other hand, developments in this area should not be exclusively focused on ICT (and related technologies, e.g. sensors, intelligent home appliances, service robotics), but need to also consider the design and launching of adequate policy actions in order to guarantee the success of any such development. Complementarily, training actions, not only for the senior citizens, but for all the other stakeholders, are a condition for success.

This trend was clearly confirmed by the BRAID roadmapping project [11], [12]. This European initiative went through an extensive consultation of stakeholders in the AAL area towards identifying the most relevant research actions in this sector for the next decade.

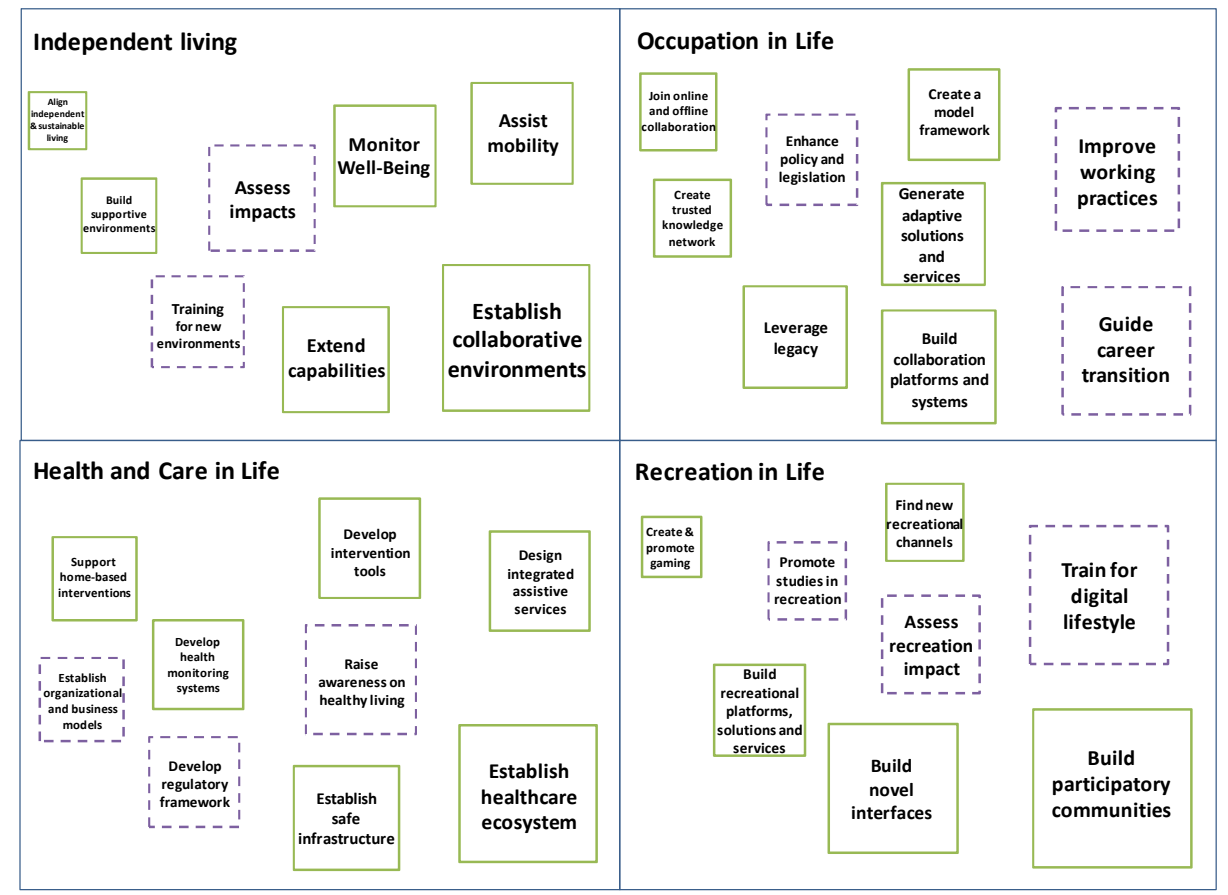

Fig. 1. Prioritization of research actions in BRAID roadmap 
When asked to prioritize the identified actions, participants in the roadmapping process clearly privileged actions such as:

- Establishment of collaborative environments for independent living

- Establishment of healthcare ecosystems

- Building collaboration platforms and systems for occupation in life

- Building participatory communities for recreation in life

- etc.

These priorities confirm the mentioned trend towards integrated services provided through collaborative ecosystems. Fig. 1 shows these findings of BRAID. Each square represents one (needed) R\&D action; the area of the squares is proportional to the number of votes given by participating stakeholders. In addition to the technology-oriented development actions, BRAID also identified the need to develop, at the same time, a number of policy related actions (dashed boxes in Fig. 1).

\section{A Conceptual Architecture}

AAL4ALL takes into account the findings and recommendations of BRAID roadmap, while adapting them to a national context. As an important element to facilitate the creation of synergies among stakeholders, a conceptual architecture was designed. This architecture aims at structuring the developments for AAL by defining a unified terminology, and describing the functionality and roles of components.

A services ecosystem model is considered in which the basic idea is to have an environment that facilitates rapid composition of (eventually multi-stakeholder) services, forming integrated care and assistance services (analogous to consortia formation). This requires that services and their providers are prepared to collaborate with each other. While designing this architecture, a socio-technical approach was followed, since socio-organizational aspects are vital to realize the potential benefits of technology in support of the ageing population. Similar to a virtual organizations breeding environment (VBE), we can consider in this environment the existence of supporting entities that take care of issues such as quality of service, billing, etc.

In order to facilitate understanding and better inter-relate the involved concepts, a 3-layered model is adopted for the AAL4ALL conceptual architecture, as illustrated in Fig. 2. Each layer is focused on specific aspects of the intended ambient assisted living environment, and a logical hierarchical structure is established among these layers.

The lowest level - the Support Infrastructure - represents a facilitator (providing support) for the development and delivery of care and assistance services. Such infrastructure should provide, among other functionalities, channels and mechanisms for safe communications and information sharing and exchange among the members of a given AAL ecosystem. As a "support" component, the infrastructure is neutral regarding any specific set of care and assistance services, or any specific organizational model of the ecosystem. The infrastructure comprises two sub-layers (Fig. 3): (1) Local infrastructure, corresponding to the support infrastructure located 
in a specific "location", e.g. user's home, care center. (2) Global infrastructure, supporting the network of "spaces" (or local environments) "inhabited" by the various stakeholders. This division is justified both by the different technical specificities of each sub-layer and (possibly) different business models associated to each one.

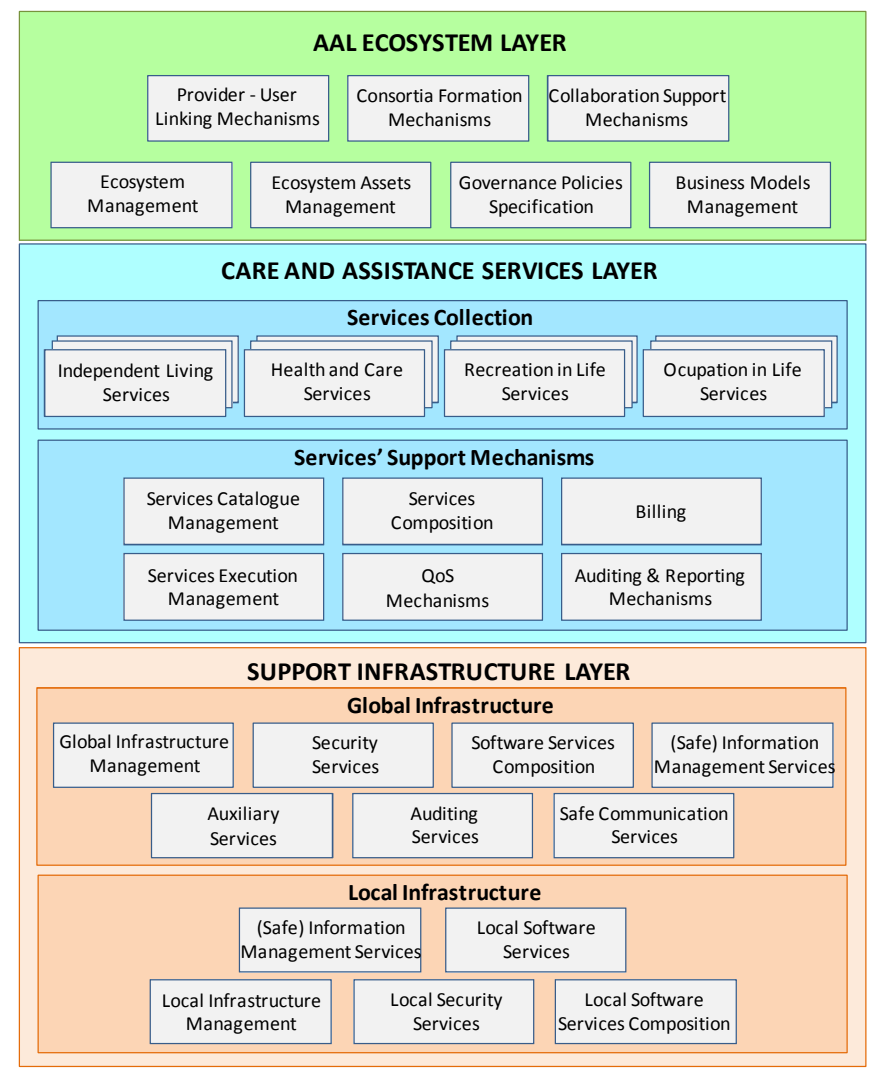

Fig. 2. Detailed diagram of the AAL4ALL architecture

The local infrastructure provides support for the user care services in his/her current location. It should allow the installation of sensors and actuators through adequate network standards. Examples of locals are the senior's home, senior hotels, care-center, senior in movement outside, and intelligent built environments.

The local infrastructure supports critical services, processes and data, requiring high level security. It will manage multiple networked sensors and actuators of several kinds, including implantable/wearable devices, as well as automation and robotic mechanisms. All these devices are modeled / wrapped as software service entities. In this sense, the local (physical) infrastructure is transformed into a software services ecosystem (which is distinct from the concept of AAL ecosystem). Main functional blocks at this level include: (i) Local Infrastructure management, (ii) Local 
security services, (iii) Local software services composition, (iv) Safe information management services, and (v) Local software services.

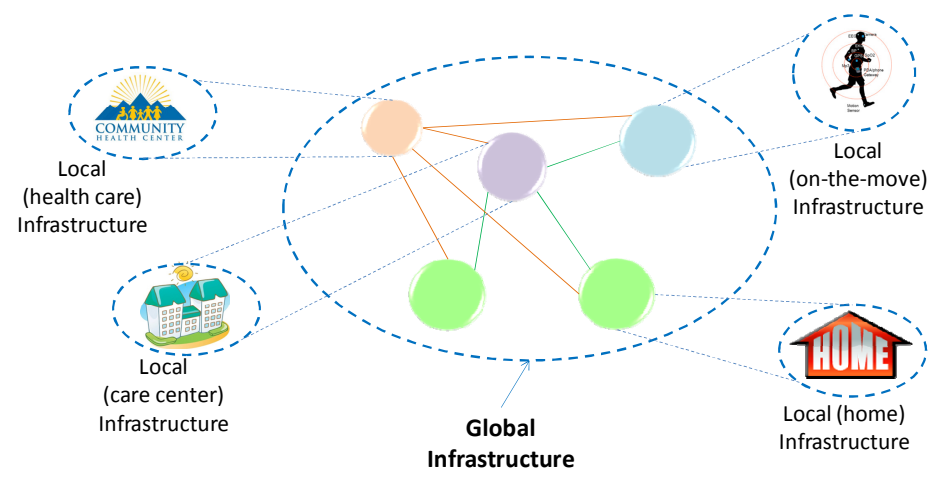

Fig. 3. Local and global infrastructures

The global infrastructure supports the interaction between the entities/nodes engaged in care provision. It supports multi-node services, distributed processes, software services invocation and composition. It can be based on a dedicated portal or on a Cloud Computing approach. Main functional blocks include: (i) Global infrastructure management, (ii) Security services, (iii) Software services composition, (iv) Safe information management services at global level, (v) Auditing services, (vi) Safe communication services, and (vii) Auxiliary services (including identification of critical issues, assessing performance, statistics and reporting).

The intermediate layer - Care and Assistance Services - provides functionalities for managing and making available an open collection of care and assistance services. The notion of "open" collection of services means that it is dynamic in the sense that services can be easily introduced, editable, replaced and removed. Functionalities allowing the construction of new and more complex services from the available elementary (atomic) services are also possible and envisioned in this layer.

In AAL4ALL a number of demonstrative services are being developed, addressing relevant needs as identified through scenarios analysis, complemented with requirements derived from an extensive set of questionnaires used to identify user needs.

This layer is logically split in two sub-layers: Services collection and Services' Support Mechanisms. The higher level represents the open care and assistance services collection. To facilitate the organization and management of the collection, care and assistance services are divided into four groups according to the four life settings of:

- Independent living - how technology can assist in normal daily life activities e.g. tasks at home, mobility, safety, agenda management (memory help), etc.

- Health and care in life - how technology can assist in health monitoring, disease prevention, and compensation for disabilities. 
- Occupation in life - how technology can support the continuation of professional activities along the ageing process.

- Recreation in life - how technology can facilitate socialization and participation in leisure activities.

The lower level layer comprises a set of support mechanisms for the management of the services. Main functional elements include: (i) Services catalog management, (ii) Services composition mechanisms, (iii) Billing, (iv) Services execution management, (v) QoS Mechanisms, and (vi) Auditing and reporting mechanisms.

The top layer of the architecture - AAL Ecosystem - provides organization, governance, and collaboration support for the AAL multi-stakeholders from a sociotechnical perspective.

An AAL ecosystem can involve, in addition to the senior citizens, a combination of formal care and informal care networks, as illustrated in Fig. 4.

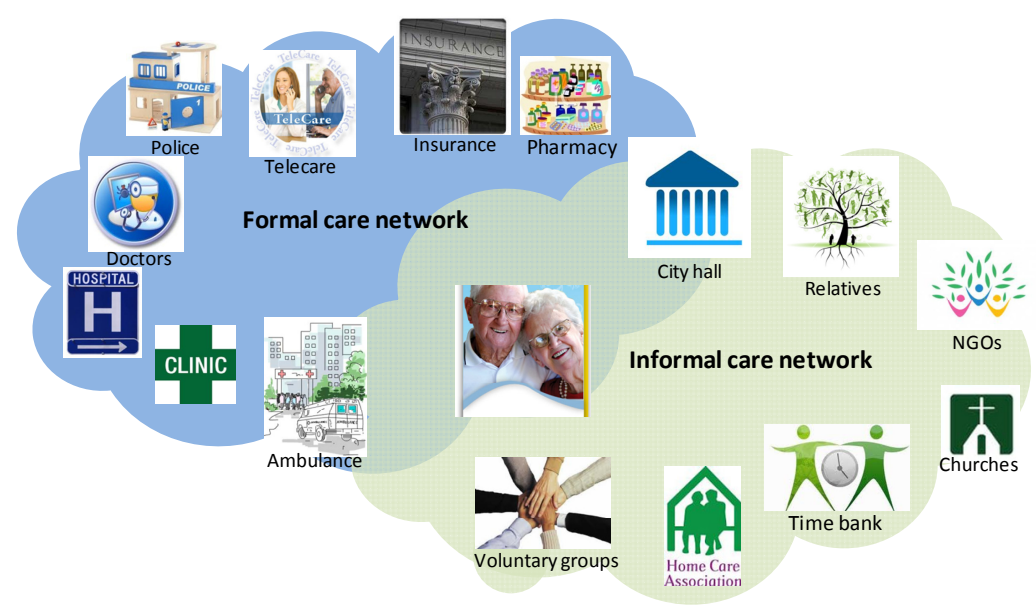

Fig. 4. Example of AAL ecosystem

The purposes of the AAL Ecosystem can only be achieved if adequate functionalities for modeling and management are provided. Such functionalities should then give support to organize and structure dynamic organizations; defining and enforcing governance policies; defining profiles, roles, business models, launching collaborative processes, and supporting links between providers and clients/users. Main functional elements of this layer include:

(i) Ecosystem Management - To provide effective management of the AAL Ecosystem in terms of service providers, users, regulators, and support entities. Functionalities for membership and roles management; profiles and competencies management (providers); user profiles management; other stakeholders management, and management of interaction with external entities. It also includes a model of the organizational structure. 
(ii) Assets Management - To provide mechanisms that allow the management of all AAL Ecosystem assets: products, services, shared knowledge, etc. It includes modeling of assets, and their ownership and access rights as well as mechanisms for sharing of assets, as well as market gap analysis.

(iii) Governance Policies Specification - To provide mechanisms that allow the specification of the governance policies of the AAL Ecosystem, including collaboration agreements. It includes definition of governance policies through instantiation of templates, rules / clauses, etc., as well as definition of rights and duties and identification of performance indicators.

(iv) Business Models Management - To provide means for identification, characterization and management of specific business models adopted by the AAL Ecosystem and its members. Such mechanisms include: the specification of the business models, contracting, accounting services, specific business plans, support mechanisms, etc. Associated to the business models, value systems are also modeled. Models for assignment of responsibilities / liabilities and benefits distribution are included. This element also includes the definition of service packages tailored to each user / class of users.

(v) Providers - Users Linking Mechanisms - To provide mechanisms to support links between AAL services or products providers and end users of the AAL Ecosystem. In other words, offering mechanisms to promote usage of the care and assistance services offered by the AAL ecosystem. A variety of mechanisms can be considered in each ecosystem, including: e-Marketplace, Brokerage, Dissemination and marketing, etc.

(vi) Consortia Formation Mechanisms - To provide mechanisms that allow (rapid) consortia formation among AAL providers, including external entities if needed, in order to deliver integrated services. It also includes consortia formation mechanisms in response to emergency situations, selection criteria specific to each ecosystem and involving elements such as stakeholders' profiles / offered services, past record of QoS, availability, collaboration readiness, costs, etc.

(vii) Collaboration Support Mechanisms - To provide mechanisms to support cooperation and/or collaboration among the AAL Ecosystem members. A collaboration platform allowing multiple collaboration processes, involving different subsets of stakeholders. Therefore, different virtual collaboration spaces should be allowed.

\section{$4 \quad$ Implementation Issues}

Implementation Architectures. As mentioned above, the purpose for the AAL4ALL conceptual architecture is to provide a kind of reference framework for the various stakeholders in the sector. Particular implementations will require the derivation / instantiation of implementation architectures that detail the intended systems and give guidance on how to implement them. In order to validate the conceptual framework, which was already the result of wide consultation among stakeholders, AAL4ALL is 
currently implementing a number of pilot cases (large scale trial), covering an extensive set of scenarios.

The feasibility of sustainable AAL ecosystems supported by an environment developed according to the concepts of the proposed architecture depends on the elaboration of appropriate business models that go "hand-in-hand" with the technoorganizational developments. Therefore, a number of critical questions related to the business models are also being addressed: Who pays for / who owns the infrastructure? Which business model for implementations based on cloud computing? Which service billing criteria? Which value systems and benefits distribution model? Etc.

AAL Ecosystem: Regional or national? One of the characteristics of Internet and computer networks in general is to allow some independence from geographical barriers. This characteristic allows remote delivery of care and assistance services, what could suggest the possibility of building an AAL ecosystem at national (if not European) level. On the other hand we cannot ignore the reality of existing organizational structures - many entities operate on a regional / local basis, e.g. care centers, health care centers, city hall related entities, etc. Furthermore, the importance of local communities in the process of supporting social inclusion of senior citizens is well recognized. Therefore, it seems more realistic to focus on regional / local AAL ecosystems. Even within one (small) geographical area we might foresee the emergence of different AAL ecosystems based on different criteria (e.g. cultural, interests, economic level).

Certainly there are major stakeholders (e.g. infrastructure operators, special service providers, insurance companies, etc.) that operate at national (or international) level. But this fact is not an obstacle for a model based on local ecosystems, since such stakeholders might participate in several local ecosystems.

The notion of local ecosystem, although associated to a community present in a given geographical area, is not strictly bounded by geographical borders. For instance, relatives of senior citizens might be living in different geographical regions and still be part (mostly through remote access) of a local ecosystem where their senior relatives live.

Nevertheless, although AAL4ALL can foresee a future scenario in which care and assistance to elderly is provided through a multiplicity of local ecosystems, there are clear advantages, from a perspective of economy of scale that all these local ecosystems are built following a common conceptual architecture (a kind of reference architecture at national level). Some form of federation of those ecosystems would also be useful to allow more affordable access to some specific services (e.g. very specialized health care services) and also to guarantee continuity of services when users travel from one region to another (a kind of "roaming between ecosystems").

\section{Conclusions}

ICT combined with new collaborative organizational structures represent a promising contribution to face the challenges of providing care and assistance services to a 
rapidly growing percentage of aged population. In this direction, many efforts have been carried out during last decade, but most of them were focused on the development of single, non-integrated services. Current trends point to the need of more integrated services, which are likely to result from contributions of various stakeholders.

In this context, the AAL4ALL project has developed a conceptual architecture to support an ecosystem of integrated (collaborative) services. The architecture follows a holistic socio-technical approach, which is reflected in the ecosystem notion, in opposition to more traditional techno-centric solutions. This proposal is aimed at acting as a facilitator for the necessary "convergence" of stakeholders and effective support for their collaboration. Having a technology-independent conceptual architecture facilitates evolution and coping with emerging technologies. The set of technology / service developers that adhere to a common conceptual architecture can more easily collaborate in specific ecosystems (shorter adaptation time), which represents a competitive advantage in comparison with outsiders.

Acknowledgments. This work was funded in part by the Project AAL4ALL (QREN 13852), co-financed by the European Community Fund through COMPETE Programa Operacional Factores de Competitividade. Partial support was also obtained from the European Commission through the BRAID project (FP7 programme). The authors also thank the contributions from their partners in these projects.

\section{References}

1. Aguilar, J.M., Cantos, J., Exposito, G., Gómez, P.: The improvement of the quality of life for elderly and relatives through two tele-assistance services: The TeleCARE approach. In: Proceedings of TELECARE 2004 Workshop - Tele-Care and Collaborative Virtual Communities, pp. 73-85. INSTICC Press, Porto (2004)

2. Camarinha-Matos, L.M., Rosas, J., Oliveira, A.: A mobile agents platform for telecare and teleassistance. In: Proceedings of TELECARE 2004 - Int. Workshop on Tele-Care and Collaborative Virtual Communities in Elderly Care, pp. 37-48. INSTICC Press, Porto (2004)

3. Costa, R., Novais, P., Costa, Â., Neves, J.: Memory Support in Ambient Assisted Living. In: Camarinha-Matos, L.M., Paraskakis, I., Afsarmanesh, H. (eds.) PRO-VE 2009. IFIP AICT, vol. 307, pp. 745-752. Springer, Heidelberg (2009)

4. Vontas, A., Protogeros, N., Moumtzi, V.: Practices and Services for Enabling the Independent Living of Elderly Population. In: Camarinha-Matos, L.M., Paraskakis, I., Afsarmanesh, H. (eds.) PRO-VE 2009. IFIP AICT, vol. 307, pp. 753-758. Springer, Heidelberg (2009)

5. USDHHS, Active Aging: A Shift in the Paradigm - Denver Summit of Eight (Industrial Countries). U.S. Department of Health and Human Services (May 1997),

http: / / aspe.hhs.gov/daltcp/reports/actaging.pdf

6. Garlick, S., Soar, J.: Human capital, innovation and the productive ageing: Growth and senior aged health in the regional community through engaged higher education. In: Annual AUCEA Conference, Alice Springs, Australia, July 2-4 (2007)

7. Llmarinen, J.: Aging and Work Life Balance in the EU (June 2006) 
8. HSBC Insurance, The future of retirement - The new old age (May 2007), http://www.hsbc.com/1/PA_1_1_S5/content/assets/retirement/ gender_perspective_eurasia_africa_1.pdf

9. Franco, R.D., Bas, Á.O., Prats, G., Varela, R.N.: Supporting Structural and Functional Collaborative Networked Organizations Modeling with Service Entities. In: CamarinhaMatos, L.M., Paraskakis, I., Afsarmanesh, H. (eds.) PRO-VE 2009. IFIP AICT, vol. 307, pp. 547-554. Springer, Heidelberg (2009)

10. Cardoso, T., Camarinha-Matos, L.M.: Pro-activity in Collaborative Service Ecosystems. In: Camarinha-Matos, L.M., Pereira-Klen, A., Afsarmanesh, H. (eds.) PRO-VE 2011. IFIP AICT, vol. 362, pp. 377-387. Springer, Heidelberg (2011)

11. Camarinha-Matos, L.M., Afsarmanesh, H.: Collaborative Ecosystems in Ageing Support. In: Camarinha-Matos, L.M., Pereira-Klen, A., Afsarmanesh, H. (eds.) PRO-VE 2011. IFIP AICT, vol. 362, pp. 177-188. Springer, Heidelberg (2011)

12. Camarinha-Matos, L.M., Ferrada, F., Oliveira, A.I., Rosas, J.: Consolidated roadmap for ICT and Ageing, Deliverable D6.21, BRAID project (October 2011), http: / /www. braidproject.eu/ 